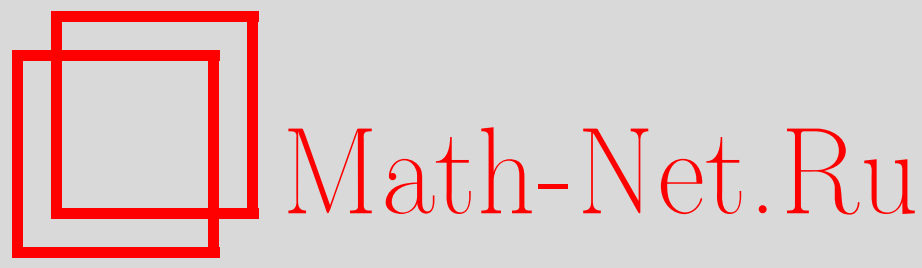

В. Н. Соловьев, К теории минимаксно-байесовского оценивания, Теория вероятн. и ее примен., 1999, том 44, выпуск 4, 738-756

DOI: https://doi.org/10.4213/tvp1063

Использование Общероссийского математического портала Math-Net.Ru подразумевает, что вы прочитали и согласны с пользовательским соглашением

http://www . mathnet.ru/rus/agreement

Параметры загрузки:

IP : 3.85 .183 .62

26 апреля 2023 г., 05:42:35

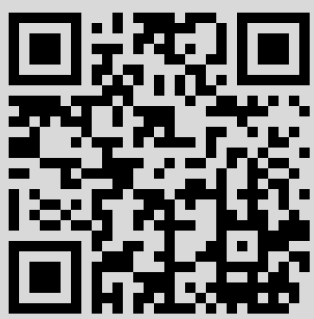




\title{
К ТЕОРИИ МИНИМАКСНО-БАЙЕСОВСКОГО ОЦЕНИВАНИЯ ${ }^{1)}$
}

\begin{abstract}
Получены обобщения теоремы о нормальной корреляции, устанавливающие линейность минимаксно-байесовских оценок и нормальность наименее благоприятных распределений. Даны приложения к оцениванию параметров в линейных моделях наблюдения.

Ключевые слова и фразы: минимаксное оценивание, двойственная задача, ковариационная матрица, нормальное распределение.
\end{abstract}

Введение. Различные постановки задач минимаксного оценивания были независимо и примерно в одно и то же время предложены А. Н. Колмогоровым, Н.Н. Красовским, М.Л. Лидовым и П. Хьюбером (см. библиографию в [9]). Одна из первых таких задач была поставлена А.Н. Колмогоровым в 1963 г. в связи с исследованиями солнечной активности. В предложенной им модели наблюдений предполагалось, что измерения $\gamma\left(t_{j}\right)=f\left(t_{j}\right)+\varepsilon_{j}, \mathbf{E} \varepsilon_{j}=0$, содержат тренд $f(t)$ с непрерывной производной $\left|f^{(m)}(t)\right| \leqslant$ const. Заменяя тренд $f(t)$ его тейлоровским разложением и затем включая последний член этого разложения в суммарные ошибки $\xi_{j}$, получим, что $\gamma\left(t_{j}\right)=\sum_{k=0}^{m-1} \theta_{k} t_{j}^{k} / k !+\xi_{j}$, где $\theta_{k}=f^{(k)}(0)$ и суммарные ошибки $\xi_{j}$ имеют ограниченные первые моменты: $\left|\mathbf{E} \xi_{j}\right| \leqslant$ const $\left|t_{j}\right|^{m} / m$ !. Из других соображений к весьма близкой задаче пришел М.Л. Лидов, который предполагал, что ошибки являются суммой белого шума и произвольно коррелированной помехи (в этом случае получаются суммарные ошибки с ограниченными вторыми моментами).

В п. 1 вводятся классы распределений случайного вектора $Y=(\gamma, \theta)$ с ограниченными вторыми моментами. В п. 2 получены обобщения теоремы о нормальной корреляции, в которых установлена линейность минимаксно-байесовских оценок и нормальность наименее благоприятных распределений. В п. 3 рассмотрены минимаксно-байесовские оценки параметров в линейных моделях наблюдения $\gamma=A \theta+\rho$, в которых

${ }^{*}$ Московский государственный социальный университет; e-mail: solovio@orc.ru

1) Работа выполнена при поддержке Российского фонда фундаментальных исследований, грант № 97-01-01005. 
вектор случайных параметров $\theta$ некоррелирован с вектором ошибок $\rho$, а ковариационные матрицы этих векторов принадлежат априорно заданным классам. Самым простым априорным ограничением на параметры служит точность $\mathbf{E}\left|\theta-\theta_{0}\right|^{2} \leqslant \sigma^{2}$ априорной оценки $\mathbf{E} \theta=\theta_{0}$ параметров $\theta$. Оказывается, что если оценивать весь вектор параметров, то при $\theta_{0}=0$ минимаксно-байесовская оценка является простым сжатием МНК-оценки. В п. 4 рассмотрены минимаксно-байесовские оценки параметров $\theta$ в линейной модели наблюдений $\gamma=A \theta+\rho, \mathbf{E} \theta=\theta_{0}, \mathbf{E} \rho=0$ в той крайней ситуации, когда нам ничего не известно о взаимной ковариации векторов $\theta$ и $\rho$ и мы ожидаем самого худшего. Оказалось, что если оценивать весь вектор параметров, то для получения минимаксной оценки нужно по одним направлениям взять МНК-оценки параметров, а по остальным довольствоваться их априорными значениями. В п. 5 доказаны вспомогательные результаты.

1. Постановка задачи. Обозначим через $\mathscr{P}=\mathscr{P}_{0}$ класс всех распределений $P(y)$ случайного вектора $Y=(\gamma, \theta)$ с компонентами $\gamma \in \mathbf{R}^{n}$, $\theta \in \mathbf{R}^{m}$, имеющего известное среднее $\mathbf{E}_{P} Y=Y_{0}=\left(\gamma_{0}, \theta_{0}\right)$, но неизвестную матрицу ковариаций $K=\operatorname{cov}(Y, Y)$, которая принадлежит априорно заданному классу $\mathscr{K}$ неотрицательно определенных матриц порядка $n+m$. Пусть по наблюдениям компоненты $\gamma$ нужно найти оценку $\hat{l}=\hat{l}(\gamma)$ для вектора $l=C^{\mathrm{T}} \theta \in \mathbf{R}^{r}$. В качестве характеристики точности этой оценки примем величину

$$
D(\hat{l}):=\sup _{P \in \mathscr{P}} \mathbf{E}_{P}|\hat{l}-l|^{2} .
$$

Для отыскания минимаксно-байесовской оценки $\hat{l}_{*}(\gamma)$ нужно на классе всех измеримых функций $\hat{l}(\gamma): \mathbf{R}^{n} \rightarrow \mathbf{R}^{r}$ найти решение задачи

$$
D_{\min }:=\inf _{\hat{l}(\cdot)} D(\hat{l}) \text {. }
$$

Мы покажем, что в минимаксной задаче (1.1)-(1.2) существует седловая точка $\left(\hat{l}_{*}, P_{*}\right)$, и поэтому она эквивалентна двойственной

$$
D_{\min }=\sup _{P \in \mathscr{P}} \inf _{\hat{l}(\cdot)} \mathbf{E}_{P}|\hat{l}-l|^{2} .
$$

Распределение $P_{*} \in \mathscr{P}$, которое доставляет максимум в (1.3), мы будем называть наименее благоприятным. Введем обозначения

$$
K=\left(\begin{array}{ll}
K_{\gamma \gamma} & K_{\gamma \theta} \\
K_{\theta \gamma} & K_{\theta \theta}
\end{array}\right), \quad \text { где } \begin{aligned}
& K_{\gamma \gamma}=\operatorname{cov}(\gamma, \gamma) \\
& K_{\gamma \theta}=\operatorname{cov}(\gamma, \theta)
\end{aligned} ;
$$

$\mathrm{Sp} K-$ след матрицы $K ;\langle A, B\rangle=\operatorname{Sp} A B^{\mathrm{T}}-$ скалярное произведение матриц $A$ и $B ; K^{+}-$псевдообратная матрица. 


\section{2. Линейность минимаксно-байесовских оценок.}

Теорема 2.1. Если множество $\mathscr{K}$ компактно и выпукло, то

$$
D_{\min }=\max _{K \in \mathscr{K}}\left\langle\left(K_{\theta \theta}-K_{\theta \gamma} K_{\gamma \gamma}^{+} K_{\gamma \theta}\right) C, C\right\rangle .
$$

Наименее благоприятным в априорном классе $\mathscr{P}_{0}$ является нормальное распределение $Y \sim \mathbb{N}\left(Y_{0}, \bar{K}\right)$, в котором $\bar{K} \in \mathscr{K}$ - любое решение задачи (2.1). Решение исходной задачи (1.1)-(1.2) дает линейная оченка $\hat{l}_{*}$, которая в случае невырожденной матричы $\bar{K}_{\gamma \gamma}$ имеет вид

$$
\hat{l}_{*}=C^{\mathrm{T}}\left(\theta_{0}+\bar{K}_{\theta \gamma} \bar{K}_{\gamma \gamma}^{-1}\left(\gamma-\gamma_{0}\right)\right) .
$$

Доказат ельст во. Сначала рассмотрим случай $l=\theta$ (т.е. $\left.C=I_{m}\right)$. Решим задачу сперва для класса линейных оценок $\hat{\theta}=L^{\mathrm{T}} \gamma+q$, $L \in \mathbf{R}^{n m}, q \in \mathbf{R}^{m}$ :

$$
\widetilde{D}_{\min }=\inf _{\hat{\theta}=L^{\mathrm{T}} \gamma+q} \sup _{P \in \mathscr{P}} \mathbf{E}_{P}|\hat{\theta}-\theta|^{2}
$$

Для линейных оценок величина $\mathbf{E}_{P}|\hat{\theta}-\theta|^{2}=\langle K X, X\rangle+\mid q+$ $\left.X^{\mathrm{T}} Y_{0}\right|^{2}, \quad X^{\mathrm{T}}:=\left(L^{\mathrm{T}},-I_{m}\right)$, зависит от распределения $P$ только через соответствующую матрицу ковариаций $K=\mathbf{E}_{P}\left(Y-Y_{0}\right)\left(Y-Y_{0}\right)^{\mathrm{T}}$. Кроме того, она линейна по переменной $K$ на выпуклом компакте $\mathscr{K}$, и выпукла по переменным $X$ и $q$ в совокупности на аффинном подпространстве. Поэтому по несимметричной теореме о минимаксе [4]

$$
\widetilde{D}_{\min }=\sup _{P \in \mathscr{P}} \inf _{\hat{\theta}=L^{\mathrm{T}} \gamma+q} \mathbf{E}_{P}|\hat{\theta}-\theta|^{2} .
$$

Для фиксированного распределения $P \in \mathscr{P}$ нижняя грань по $\hat{\theta}$ достигается на оценке

$$
\hat{\theta}=\theta_{0}+K_{\theta \gamma} K_{\gamma \gamma}^{+}\left(\gamma-\gamma_{0}\right)
$$

и равна

$$
\inf _{\hat{\theta}=L^{\mathrm{T}} \gamma+q} \mathbf{E}_{P}|\hat{\theta}-\theta|^{2}=\operatorname{Sp}\left(K_{\theta \theta}-K_{\theta \gamma} K_{\gamma \gamma}^{+} K_{\gamma \theta}\right) .
$$

Для дальнейшего сушественно, что оценка (2.5) дает единственное решение задачи (2.6), если матрица $K_{\gamma \gamma}$ невырожденна. Действительно, очевидно, что оптимален выбор вектора

$$
q=-X^{\mathrm{T}} Y_{0}=\theta_{0}-L^{\mathrm{T}} \gamma_{0}
$$

а поскольку градиент функции $\mathbf{E}_{P}|\hat{\theta}-\theta|^{2}=\operatorname{Sp} K_{\theta \theta}-2\left\langle L, K_{\gamma \theta}\right\rangle+\left\langle K_{\gamma \gamma} L, L\right\rangle$ по переменной $L \in \mathbf{R}^{n m}$ равен $2\left(K_{\gamma \gamma} L-K_{\gamma \theta}\right)$, то ее единственная точка минимума - это $L=K_{\gamma \gamma}^{-1} K_{\gamma \theta}$, что дает соотношения $(2.5)$ и (2.6) в 
случае невырожденной матрицы $K_{\gamma \gamma}$. Общий случай рассматривается аналогично.

В силу (2.4), (2.6) получаем

$$
\widetilde{D}_{\min }=\sup _{K \in \mathscr{K}} \operatorname{Sp}\left(K_{\theta \theta}-K_{\theta \gamma} K_{\gamma \gamma}^{+} K_{\gamma \theta}\right) .
$$

Как минимум семейства линейных по переменной $K$ величин $\mathbf{E}_{P}|\hat{\theta}-\theta|^{2}$, функция (2.8) вогнута и полунепрерывна сверху по переменной $K$ на выпуклом компакте $\mathscr{K}$, поэтому задача (2.8) имеет решение $\bar{K} \in \mathscr{K}$ [4]. Тем самым, и двойственная задача (2.4) имеет решение $P_{*}=\mathbb{N}\left(Y_{0}, \bar{K}\right)$.

Покажем, что исходная задача (2.3) тоже имеет решение $\hat{\theta}_{*}$. Очевидно, что оптимален выбор вектора (2.7). Тогда

$$
\widetilde{D}_{\min }=\inf \left\{D(X): X^{\mathrm{T}}=\left(L^{\mathrm{T}},-I_{m}\right) \in \mathbf{R}^{m(n+m)}\right\},
$$

где функция $D(X):=\max _{K \in \mathscr{K}}\langle K X, X\rangle$ однородна степени 2 , выпукла и неотрицательна. Поэтому она является квадратом некоторой полунормы [6], и указанная задача эквивалентна минимизации этой полунормы на аффинном подпространстве $\mathscr{L}$ в $\mathbf{R}^{(n+m) m}$. Нетрудно сообразить, что она имеет решение (так как всякая полунорма - это норма, заданная на линейном подпространстве, то можно искать минимум этой нормы на проекции аффинного подпространства $\mathscr{L}$ на это линейное подпространство). Поэтому задача (2.3) тоже имеет решение.

Таким образом, мы доказали, что двойственные задачи (2.3), (2.4) имеют решения $\hat{\theta}_{*}, P_{*}$, и значения этих задач совпадают. Отсюда следует, что $\left(\hat{\theta}_{*}, P_{*}\right)$ - седловая точка в задаче $(2.3)$ (см. $[10$, с. 172$\left.]\right)$, т.е. для всех линейных оценок $\hat{\theta}$ и распределений $P \in \mathscr{P}$ имеют место неравенства

$$
\mathbf{E}_{P}\left|\hat{\theta}_{*}-\theta\right|^{2} \leqslant \mathbf{E}_{P_{*}}\left|\hat{\theta}_{*}-\theta\right|^{2} \leqslant \mathbf{E}_{P_{*}}|\hat{\theta}-\theta|^{2} .
$$

Поскольку $\hat{\theta}_{*}$ - решение задачи $(2.6)$, которое для невырожденной матрицы $\bar{K}_{\gamma \gamma}$ единственно, то в этом случае $\hat{\theta}_{*}$ имеет вид $(2.2)$.

Теперь рассмотрим задачу (1.1)-(1.2) для класса произвольных измеримых функций $\hat{\theta}(\cdot)$. По теореме о нормальной корреляции [1], [3] в действительности правое неравенство в (2.9) выполняется для любой нелинейной оценки $\hat{\theta}$, т.е. $\left(\hat{\theta}_{*}, P_{*}\right)$ - седловая точка в исходной задаче (1.1)-(1.2). Отсюда следует, что линейная оценка $\hat{\theta}_{*}$ дает решение задачи (1.1)-(1.2) и равенство (2.1).

Наконец, рассмотрим произвольный оцениваемый параметр $l=$ $C^{\mathrm{T}} \theta \in \mathbf{R}^{r}$. Случайный вектор $\tilde{Y}=(\gamma, l)$ имеет произвольное распределение, удовлетворяющее ограничениям

$$
\mathbf{E}_{P} \tilde{Y}=\left(\gamma_{0}, l_{0}\right), \quad l_{0}:=C^{\mathrm{T}} \theta_{0}, \quad \operatorname{cov}(\tilde{Y}, \tilde{Y})=\left(\begin{array}{cc}
K_{\gamma \gamma} & K_{\gamma \theta} C \\
C^{\mathrm{T}} K_{\theta \gamma} & C^{\mathrm{T}} K_{\theta \theta} C
\end{array}\right)
$$


Поэтому для него выполнены все условия теоремы 2.1, доказанной выше для случая $l=\theta$, что с учетом $(2.8),(2.5)$ и $(2.10)$ дает равенства $(2.1)$ и (2.2). Теорема 2.1 доказана.

Теорема 2.1 является обобщением теоремы о нормальной корреляции $[1],[3]$, в котором мы использовали идеи замечательной работы [16]. В случае, когда все распределения $P \in \mathscr{P}_{0}$ имеют известные среднее $Y_{0}$ и матрицу ковариаций $K$, этот результат получен в [5]. Пользуясь методами теории двойственных экстремальных задач, можно распространить его на общий случай распределений, первые и вторые моменты которых лежат в априорно заданных классах [7]-[9], [15]-[17]. Близкие задачи рассмотрены в [11], [12].

Теорема 2.2. Если множество $\mathscr{K}$ компактно и содержит неотричательно определенные матричы, то все утверждения теоремы 2.1 останутся справедливы, если выпукло не само это множество, а матричное множество $\{\widetilde{K}: \exists K \in \mathscr{K}, K \geqslant \widetilde{K}\}$.

Д о к а з а т е л ь с т в о. Обозначим через $\mathscr{P}=\widetilde{\mathscr{P}}_{0}$ класс всех распределений $P(y)$ случайного вектора $Y$, имеющего известное среднее $\mathbf{E}_{P} Y=Y_{0}$, но неизвестную ковариационную матрицу $\operatorname{cov}(Y, Y) \in \widetilde{\mathscr{K}}$, где $\widetilde{\mathscr{K}}:=\{\widetilde{K} \geqslant 0: \exists K \in \mathscr{K}, K \geqslant \widetilde{K}\}$. Легко проверить, что множество $\widetilde{K}$ выпукло и компактно. Поскольку $\mathscr{K} \subset \widetilde{\mathscr{K}}$, то $\mathscr{P}_{0} \subset \widetilde{\mathscr{P}}_{0}$. Поэтому, согласно $(1.1), D(\hat{l}) \leqslant \widetilde{D}(\hat{l})$, где $\widetilde{D}(\hat{l})$ и $\widetilde{D}_{\min }$ обозначают величины $(1.1)$ и (1.2), вычисленные для класса распределений $\widetilde{\mathscr{P}}_{0}$. Из этого неравенства получаем $D_{\min } \leqslant \widetilde{D}_{\min }$. Более того, имеем (см. [10])

$$
D_{\min }:=\inf _{\hat{l}(\cdot)} \sup _{P \in \mathscr{P}} \mathbf{E}_{P}|\hat{l}-l|^{2} \geqslant \sup _{P \in \mathscr{P}} \inf _{\hat{l}(\cdot)} \mathbf{E}_{P}|\hat{l}-l|^{2} \geqslant \sup _{P \in \mathcal{N}} \inf _{\hat{l}(\cdot)} \mathbf{E}_{P}|\hat{l}-l|^{2},
$$

где $\mathcal{N}$ обозначает подкласс всех нормальных распределений из априорного класса $\mathscr{P}=\mathscr{P}_{0}$. По теореме о нормальной корреляции отсюда

$$
D_{\min } \geqslant \max _{K \in \mathscr{K}}\left\langle\left(K_{\theta \theta}-K_{\theta \gamma} K_{\gamma \gamma}^{+} K_{\gamma \theta}\right) C, C\right\rangle \text {. }
$$

С другой стороны, поскольку $\widetilde{\mathscr{K}}-$ выпуклый компакт, то по теореме 2.1 получаем

$$
\widetilde{D}_{\min }=\max _{\widetilde{K} \in \widetilde{\mathscr{K}}}\left\langle\left(\widetilde{K}_{\theta \theta}-\widetilde{K}_{\theta \gamma} \widetilde{K}_{\gamma \gamma}^{+} \widetilde{K}_{\gamma \theta}\right) C, C\right\rangle .
$$

В действительности правые части последних двух соотношений совпадают, ибо хотя множество $\mathscr{K}$ и меньше $\widetilde{\mathscr{K}}$, но в то же время для любой матрицы $\widetilde{K} \in \widetilde{\mathscr{K}}$ существует матрица $K \in \mathscr{K}$ такая, что $K \geqslant \widetilde{K}$. Легко проверить, что в этом случае

$$
\left\langle\left(K_{\theta \theta}-K_{\theta \gamma} K_{\gamma \gamma}^{+} K_{\gamma \theta}\right) C, C\right\rangle \geqslant\left\langle\left(\widetilde{K}_{\theta \theta}-\widetilde{K}_{\theta \gamma} \widetilde{K}_{\gamma \gamma}^{+} \widetilde{K}_{\gamma \theta}\right) C, C\right\rangle .
$$


Таким образом, окончательно получаем

$$
\widetilde{D}_{\min } \geqslant D_{\min } \geqslant \max _{K \in \mathscr{X}}\left\langle\left(K_{\theta \theta}-K_{\theta \gamma} K_{\gamma \gamma}^{+} K_{\gamma \theta}\right) C, C\right\rangle=\widetilde{D}_{\min },
$$

т.е. $D_{\min }=\widetilde{D}_{\min }$. Это доказывает (2.1). Поскольку $\mathscr{K} \subset \widetilde{\mathscr{K}}$, любое решение $\bar{K} \in \mathscr{K}$ в задаче (2.1) дает также и решение задачи (2.11). Пусть $\hat{l}_{*}$ - линейная оценка, дающая решение задачи (1.2) для априорного класса распределений $\widetilde{P}_{0}$, а $P_{*}$ - нормальное распределение $Y \sim \mathbb{N}\left(Y_{0}, \bar{K}\right)$. Тогда, как было доказано в теореме 2.1 , для всех нелинейных оценок $\hat{l}$ и распределений $P \in \widetilde{\mathscr{P}}_{0}$ будут выполнены неравенства $\mathbf{E}_{P}\left|\hat{l}_{*}-l\right|^{2} \leqslant \mathbf{E}_{P_{*}}\left|\hat{l}_{*}-l\right|^{2} \leqslant \mathbf{E}_{P_{*}}|\hat{l}-l|^{2}$. В частности, они будут выполнены для всех нелинейных оценок $\hat{l}$ и распределений $P \in \mathscr{P}_{0} \subset \widetilde{\mathscr{P}}_{0}$. Как мы уже заметили, это означает, что наименее благоприятным в априорном классе $\mathscr{P}_{0}$ является нормальное распределение $Y \sim \mathbb{N}\left(Y_{0}, \bar{K}\right)$ и что решение исходной задачи (1.1)-(1.2) дает линейная оценка $\hat{l}_{*}$. Теорема 2.2 доказана.

Заметим, что формально теорема 2.1 - частный случай этого результата. Другой частный случай, когда (невыпуклое) компактное множество $\mathscr{K}$ состоит из неотрицательно определенных матриц и содержит максимальный элемент (т.е. существует матрица $\bar{K} \in \mathscr{K}$ такая, что $K \leqslant \bar{K}$ при всех $K \in \mathscr{K}$ ), был указан в [16]. Очевидно, что в этом случае $\bar{K}$ и есть решение задачи (2.1).

Использованный нами прием расширения априорного класса можно применить и тогда, когда $\mathscr{K}-$ произвольное невыпуклое, компактное множество, состоящее из неотрицательно определенных матриц. В этом случае вместо исходного класса $\mathscr{P}=\mathscr{P}_{0}$ можно рассмотреть более широкий класс $\mathscr{P}=\widetilde{\mathscr{P}}_{0}$ всех распределений $P(y)$ вектора $Y$, имеющих известное среднее $\mathbf{E}_{P} Y=Y_{0}$ и ковариационную матрицу $\operatorname{cov}(Y, Y) \in \widetilde{\mathscr{K}}$, где $\widetilde{\mathscr{K}}:=\operatorname{conv} \mathscr{K}-$ выпуклая оболочка множества $\mathscr{K}$. На этом более широком классе распределений минимаксно-байесовская оценка уже будет линейной. Поэтому в условиях весьма значительной неопределенности статистических характеристик распределения $P$ можно смело рекомендовать использование линейных оценок, как из соображений их большей устойчивости, так и из соображений их несомненной простоты.

\section{3. Минимаксно-байесовские оценки параметров в линей-} ных моделях наблюдения. Пусть в линейной модели $\gamma=A \theta+\rho$ вектор случайных параметров $\theta$ некоррелирован с вектором ошибок $\rho$, а ковариационные матрицы этих векторов принадлежат априорным классам $\mathscr{T}$ и $\mathscr{R}:$

$$
\begin{gathered}
\mathbf{E} \theta=\theta_{0}, \quad \mathbf{E} \rho=0, \quad \operatorname{cov}(\theta, \theta)=T \in \mathscr{T} \\
\operatorname{cov}(\theta, \rho)=0, \quad \operatorname{cov}(\rho, \rho)=R \in \mathscr{R} .
\end{gathered}
$$


Теорема 3.1. Если множества $\mathscr{T}$ и $\mathscr{R}$ выпуклы и компактны $и$ содержат положительно определенные матриџы, то

$$
D_{\min }=\max _{T \in \mathscr{T}, R \in \mathscr{R}}\left\langle\left(T^{-1}+A^{\mathrm{T}} R^{-1} A\right)^{-1} C, C\right\rangle .
$$

Минимаксно-байесовской для вектора $l=C^{\mathrm{T}} \theta$ является линейная очен$\kappa a$

$$
\hat{l}_{*}=C^{\mathrm{T}}\left(\bar{T}^{-1}+A^{\mathrm{T}} \bar{R}^{-1} A\right)^{-1}\left(A^{\mathrm{T}} \bar{R}^{-1} \gamma+\bar{T}^{-1} \theta_{0}\right),
$$

в которой $\bar{T} \in \mathscr{T}, \bar{R} \in \mathscr{R}$ - любое решение задачи (3.2). При этом наименее благоприятны нормальные распределения $\theta \sim \mathbb{N}\left(\theta_{0}, \bar{T}\right)$, $\rho \sim \mathbb{N}(0, \bar{R})$.

Д ок аза т ельст в о. Для линейной модели наблюдений $\gamma=$ $A \theta+\rho$, удовлетворяющей $(3.1)$, блоки ковариационной матрицы $K=$ $\operatorname{cov}(Y, Y)$ случайного вектора $Y=(\gamma, \theta)$ имеют вид

$$
K_{\theta \theta}=T, \quad K_{\gamma \theta}=A T, \quad K_{\gamma \gamma}=A T A^{\mathrm{T}}+R .
$$

Поскольку множество $\mathscr{K}$ всех таких матриц компактно и выпукло, то в силу (2.1), (2.2) остается воспользоваться равенством $\mathbf{E} \gamma=\gamma_{0}=A \theta_{0}$ и известными тождествами (см. [14])

$$
\begin{aligned}
K_{\theta \theta}-K_{\theta \gamma} K_{\gamma \gamma}^{-1} K_{\gamma \theta} & =\left(T^{-1}+A^{\mathrm{T}} R^{-1} A\right)^{-1} \\
K_{\theta \gamma} K_{\gamma \gamma}^{-1} & =\left(T^{-1}+A^{\mathrm{T}} R^{-1} A\right)^{-1} A^{\mathrm{T}} R^{-1} .
\end{aligned}
$$

Теорема 3.1 доказана.

Самым простым априорным ограничением на параметры служит точность априорной оценки $\theta_{0}$ :

$$
\mathbf{E} \theta=\theta_{0}, \quad \mathbf{E}\left|\theta-\theta_{0}\right|^{2} \leqslant \sigma^{2} .
$$

В такой ситуации естественно оценивать весь вектор параметров $l=\theta$ на классе $\mathscr{P}_{0}$ всех распределений случайных векторов $\theta$ и $\rho$, удовлетворяющих ограничениям (3.1) и (3.6). Напомним, что если известно точное значение $R=\operatorname{cov}(\rho, \rho)$ ковариационной матрицы ошибок наблюдений $\rho$, а априорные данные о векторе параметров отсутствуют, то популярная оценка параметров получается по методу наименьших квадратов (MHK):

$$
\hat{\theta}_{1}=\left(A^{\mathrm{T}} R^{-1} A\right)^{-1} A^{\mathrm{T}} R^{-1} \gamma .
$$

Теорема 3.2. Пусть матрица $A$ имеет ранг $m$ и $\bar{R} \in \mathscr{R}$ - наименее благоприятная матрича ковариачий для $M H K$-оченок (3.7), являющаяся решением задачи

$$
\max _{R \in \mathscr{R}} \operatorname{Sp} D(R), \quad D(R):=\left(A^{\mathrm{T}} R^{-1} A\right)^{-1} .
$$


Тогда минимаксно-байесовской является линейная оченка параметров

$$
\hat{\theta}_{*}=\frac{\sigma^{2} \hat{\theta}_{1}+\operatorname{Sp} D(\bar{R}) \theta_{0}}{\sigma^{2}+\operatorname{Sp} D(\bar{R})}
$$

в которой $\hat{\theta}_{1}$ - это МНК-оченка (3.7), вычисленная для матричы $\bar{R}$. Наименее благоприятными являются нормальные распределения $\theta \sim \mathbb{N}\left(\theta_{0}, \sigma^{2} D(\bar{R}) / \operatorname{Sp} D(\bar{R})\right)$ и $\rho \sim \mathbb{N}(0, \bar{R})$, причем

$$
D_{\min }=\frac{\sigma^{2} \operatorname{Sp} D(\bar{R})}{\sigma^{2}+\operatorname{Sp} D(\bar{R})} .
$$

Д о к а з а т е л с с т в о. Сначала рассмотрим случай одноточечного множества $\mathscr{R}=\{\bar{R}\}$ и априорного множества

$$
\mathscr{T}_{\varepsilon}=\left\{T \geqslant \varepsilon I_{m}, \operatorname{Sp} T \leqslant \sigma^{2}\right\},
$$

где $\varepsilon>0$. Пусть $P_{*} \in \mathscr{P}$ будет нормальным распределением вектора $Y=(\gamma, \theta)$, указанным в теореме 3.2 . Проверим, что пара $\left(\hat{\theta}_{*}, P_{*}\right)$ образует седловую точку в задаче (1.1)-(1.2).

Чтобы показать, что $\hat{\theta}_{*}=\mathbf{E}_{P_{*}}(\theta \mid \gamma)$, воспользуемся представлением условного среднего $\hat{\theta}_{*}=\mathbf{E}_{P_{*}}(\theta \mid \gamma)$ в виде $(3.3)$ с невырожденной матрицей ковариаций

$$
\bar{T}=\frac{\sigma^{2}}{\operatorname{Sp} D(\bar{R})} D(\bar{R}) .
$$

С учетом обозначения (3.8) получаем

$$
\left(\bar{T}^{-1}+A^{\mathrm{T}} \bar{R}^{-1} A\right)^{-1}=\frac{\sigma^{2}}{\sigma^{2}+\operatorname{Sp} D(\bar{R})} D(\bar{R}) .
$$

Тогда равенство (3.3) переходит в (3.9), т.е. $\hat{\theta}_{*}=\mathbf{E}_{P_{*}}(\theta \mid \gamma)$.

Покажем, что распределение $P_{*}$ наименее благоприятно для оценки $\hat{\theta}_{*}$. Имеем

$$
\begin{aligned}
\mathbf{E}_{P}\left|\hat{\theta}_{*}-\theta\right|^{2} & =\mathbf{E}_{P}\left|\frac{\sigma^{2}}{\sigma^{2}+\operatorname{Sp} D(\bar{R})} D(\bar{R}) A^{\mathrm{T}} \bar{R}^{-1} \rho-\frac{\operatorname{Sp} D(\bar{R})}{\sigma^{2}+\operatorname{Sp} D(\bar{R})}\left(\theta-\theta_{0}\right)\right|^{2} \\
& =\left[\frac{\sigma^{2}}{\sigma^{2}+\operatorname{Sp} D(\bar{R})}\right]^{2} \operatorname{Sp} D(\bar{R})+\left[\frac{\operatorname{Sp} D(\bar{R})}{\sigma^{2}+\operatorname{Sp} D(\bar{R})}\right]^{2} \operatorname{Sp} T
\end{aligned}
$$

в силу некоррелированности векторов $\rho$ и $\theta$ и принятого соглашения $\operatorname{cov}(\rho, \rho)=\bar{R}$. Отсюда видно, что при достаточно малых $\varepsilon>0$ максимум величины (3.13) по априорным матрицам $T$, удовлетворяющим ограничениям (3.11), достигается на матрице (3.12). 
Таким образом, $\left(\hat{\theta}_{*}, P_{*}\right)$ - седловая точка, и поэтому при фиксированной матрице $R=\bar{R}$ максимум по $T \in \mathscr{T}_{\varepsilon}$ в задаче (3.2) достигается на матрице (3.12). Подставляя ее в (3.13), получим

$$
D_{\min }=\mathbf{E}_{P_{*}}\left|\hat{\theta}_{*}-\theta\right|^{2}=\frac{\sigma^{2} \operatorname{Sp} D(\bar{R})}{\sigma^{2}+\operatorname{Sp} D(\bar{R})} .
$$

Теперь заметим, что так как матричная функция (3.12) непрерывно зависит от переменной $\bar{R}$ на компакте $\mathscr{R}$, то найдется $\varepsilon_{0}>0$ такое, что $\bar{T} \in \mathscr{T}_{\varepsilon}$ сразу для всех $\bar{R} \in \mathscr{R}$ и $0<\varepsilon<\varepsilon_{0}$. В случае произвольного выпуклого компакта $\mathscr{R}$ это означает, что максимум в $(3.2)$ равен максимуму величины (3.14) по всем матрицам $\bar{R} \in \mathscr{R}$. Поскольку эта величина монотонно возрастает относительно переменной $\operatorname{Sp} D(\bar{R})$, то решение задачи (3.2) дают матрица $\bar{R}$, являющаяся решением задачи (3.8), и соответствующая ей матрица $\bar{T}$ вида (3.12). Как мы только что убедились, этой паре матриц соответствует условное среднее $\hat{\theta}_{*}=\mathbf{E}_{P_{*}}(\theta \mid \gamma)$ вида (3.9). Таким образом, соотношения (3.9), (3.10) нами доказаны для априорного множества $\mathscr{T}_{\varepsilon}$ при $0<\varepsilon<\varepsilon_{0}$.

Наконец, заметим, что поскольку $\left(\hat{\theta}_{*}, P_{*}\right)$ - седловая точка в задаче (1.1)-(1.2), то неравенство (2.9) выполняется для всех оценок $\hat{\theta}$ и распределений $P(y)$ таких, что $R \in \mathscr{R}, T \in \mathscr{T}_{\varepsilon}, 0<\varepsilon<\varepsilon_{0}$. В силу линейности величины $\mathbf{E}_{P}\left|\hat{\theta}_{*}-\theta\right|^{2}$ по переменной $T$ в действительности оно справедливо для любой матрицы $T \in \mathscr{T}_{0}=\mathscr{T}$ (см. (3.11)), т.е. $\left(\hat{\theta}_{*}, P_{*}\right)$ - седловая точка в задаче $(1.1)-(1.2)$ для исходного класса распределений, удовлетворяющих априорным ограничениям (3.6). Тогда в этой задаче наименее благоприятными являются распределения $\theta \sim \mathbb{N}\left(\theta_{0}, \sigma^{2} D(\bar{R}) / \operatorname{Sp} D(\bar{R})\right)$ и $\rho \sim \mathbb{N}(0, \bar{R})$. Теорема 3.2 доказана.

Отметим, что если известно точное значение $R=\bar{R}$ ковариационной матрицы ошибок наблюдений $\rho$, то формула (3.9) дает аналитическое выражение для минимаксно-байесовской оценки параметров, причем при $\theta_{0}=0$ эта оценка является простым сжатием МНК-оценки (3.7). Интересно, что в этом случае апостериорная точность $\mathbf{E}_{P}\left|\hat{\theta}_{*}-\theta\right|^{2}$ будет одной и той же для всех распределений $P \in \mathscr{P}_{0}$ с одинаковой величиной априорной точности $\mathbf{E}_{P}\left|\theta-\theta_{0}\right|^{2}$ и что в этом случае оценка (3.9) оптимальна в совсем иной задаче [13].

4. Минимаксно-байесовские оценки параметров в случае произвольной корреляции сигнала и шума. Пусть теперь в модели

$$
\begin{gathered}
\gamma=A \theta+\rho, \quad \mathbf{E} \theta=\theta_{0}, \quad \mathbf{E} \rho=0, \\
\operatorname{cov}(\theta, \theta):=T \in \mathscr{T}, \quad \operatorname{cov}(\rho, \rho):=R \in \mathscr{R}
\end{gathered}
$$

нам ничего не известно о взаимной ковариации $\theta$ и $\rho$, кроме неотрицательной определенности матрицы ковариаций вектора $(\theta, \rho) \in \mathbf{R}^{n+m}$, и 
мы ожидаем самого худшего. Здесь мы будем считать, что ранг матрицы $A$ равен $m$ и что $\mathscr{T}$ и $\mathscr{R}$ - выпуклые компакты положительно определенных матриц.

Теорема 4.1. Справедливо равенство

$$
D_{\min }=\max _{D \geqslant 0, T \in \mathscr{T}, R \in \mathscr{R}}\left\{\operatorname{Sp} C^{\mathrm{T}} D C: D \leqslant T, D \leqslant\left(A^{\mathrm{T}} R^{-1} A\right)^{-1}\right\} .
$$

Наименее благоприятно в задаче (1.2), (4.1) нормальное распределение вектора $(\theta, \rho)$ в случае

$$
\operatorname{cov}(\theta, \theta)=T_{*}, \quad \operatorname{cov}(\rho, \rho)=R_{*}, \quad \operatorname{cov}(\rho, \theta)=-A D_{*},
$$

где $D_{*}, T_{*}, R_{*}$ - решение задачи (4.2), а минимаксно-байесовская оченка $\hat{l}_{*}$ линейна.

Д о к а з а т е л ь с т в о. Случайный вектор $Y=(\gamma, \theta)$ с компонентами $\gamma \in \mathbf{R}^{n}, \theta \in \mathbf{R}^{m}$ имеет известное среднее $\mathbf{E} Y=Y_{0}=\left(A \theta_{0}, \theta_{0}\right)$ и неизвестную ковариационную матрицу $K=\operatorname{cov}(Y, Y)$, которая принадлежит выпуклому компакту

$$
\begin{aligned}
\mathscr{K}=\{ & K \geqslant 0: K_{\theta \theta}=T \in \mathscr{T}, K_{\gamma \gamma}-K_{\gamma \theta} A^{\mathrm{T}}-A K_{\theta \gamma} \\
& \left.+A K_{\theta \theta} A^{\mathrm{T}}=R \in \mathscr{R}\right\} .
\end{aligned}
$$

По теореме 2.1 поэтому справедливо равенство (2.1), причем оценка $\hat{l}_{*}$ будет линейна. Для любой матрицы $K \in \mathscr{K}$ фигурирующая в нем матрица

$$
D=K_{\theta \theta}-\dot{K}_{\theta \gamma} K_{\gamma \gamma}^{+} K_{\gamma \theta}
$$

удовлетворяет неравенствам $0 \leqslant D \leqslant K_{\theta \theta}=T$ (см. [1]). Кроме того, поскольку она является ковариационной матрицей оптимальной оценки (2.5) для нормального распределения $P=\mathbb{N}\left(Y_{0}, K\right)$, то

$$
D \leqslant \operatorname{cov}\left(\hat{\theta}_{1}-\theta, \hat{\theta}_{1}-\theta\right)=D(R):=\left(A^{\mathrm{T}} R^{-1} A\right)^{-1},
$$

где $\hat{\theta}_{1}$ обозначает МНК-оценку (3.7), а ее ковариационная матрица берется относительно распределения $P=\mathbb{N}\left(Y_{0}, K\right)$. Следовательно, матрица (4.5) удовлетворяет ограничениям задачи (4.2), и поэтому значение этой задачи не меньше значения задачи (2.1).

Наоборот, пусть $D$ - произвольная матрица, удовлетворяющая ограничениям задачи (4.2):

$$
0 \leqslant D \leqslant T, \quad D \leqslant D(R) .
$$

Докажем, что матрица

$$
K=\left(\begin{array}{cc}
R+A(T-2 D) A^{\mathrm{T}} & A(T-D) \\
(T-D) A^{\mathrm{T}} & T
\end{array}\right),
$$


формально соответствующая выбору $\operatorname{cov}(\rho, \theta)=-A D$, действительно является ковариационной для случайного вектора $Y$ (т.е. принадлежит матричному множеству $\mathscr{K}$, определенному в (4.4)) и удовлетворяет неравенству

$$
K_{\theta \theta}-K_{\theta \gamma} K_{\gamma \gamma}^{+} K_{\gamma \theta} \geqslant D
$$

(в этот момент $K_{\theta \theta}, K_{\theta \gamma}, K_{\gamma \gamma}$ обозначают просто блоки матрицы $K$ ).

Для этого вычислим квадратичную форму $\langle K x, x\rangle$ с вектором $x:=\left(x_{0}, x_{1}\right) \in \mathbf{R}^{n+m}$, где $x_{0} \in \mathbf{R}^{n}, x_{1} \in \mathbf{R}^{m}$. Формально она равна дисперсии случайной величины $\left\langle x_{0}, \gamma\right\rangle+\left\langle x_{1}, \theta\right\rangle=\left\langle x_{0}, \rho\right\rangle+\left\langle A^{\mathrm{T}} x_{0}+x_{1}, \theta\right\rangle$. Поэтому для любого $x \in \mathbf{R}^{n+m}$ имеем

$$
\begin{aligned}
\langle K x, x\rangle= & \left\langle R x_{0}, x_{0}\right\rangle+\left\langle T\left(A^{\mathrm{T}} x_{0}+x_{1}\right), A^{\mathrm{T}} x_{0}+x_{1}\right\rangle \\
& -2\left\langle A D\left(A^{\mathrm{T}} x_{0}+x_{1}\right), x_{0}\right\rangle \equiv\left\langle\left(R-A D A^{\mathrm{T}}\right) x_{0}, x_{0}\right\rangle \\
& +\left\langle(T-D)\left(A^{\mathrm{T}} x_{0}+x_{1}\right), A^{\mathrm{T}} x_{0}+x_{1}\right\rangle+\left\langle D x_{1}, x_{1}\right\rangle .
\end{aligned}
$$

Чтобы показать, что матрица (4.7) неотрицательно определена, мы должны проверить, что $\langle K x, x\rangle \geqslant 0$ для любого $x \in \mathbf{R}^{n+m}$.

Согласно (4.6), $T-D \geqslant 0, D \geqslant 0$, поэтому последние два слагаемых в тождестве (4.9) неотрицательны. Покажем, что и первое тоже:

$$
R-A D A^{\mathrm{T}} \geqslant 0 \text {. }
$$

В силу (4.6) $A D A^{\mathrm{T}} \leqslant A\left(A^{\mathrm{T}} R^{-1} A\right)^{-1} A^{\mathrm{T}}=R^{1 / 2} Q R^{1 / 2}$, где $Q:=$ $R^{-1 / 2} A\left(A^{\mathrm{T}} R^{-1} A\right)^{-1} A^{\mathrm{T}} R^{-1 / 2}-$ это оператор ортогонального проектирования $\left(Q^{\mathrm{T}}=Q, Q^{2}=Q\right)$. Поскольку $Q \leqslant I_{n}$, то $R^{1 / 2} Q R^{1 / 2} \leqslant$ $R^{1 / 2} R^{1 / 2}=R$, что с учетом предыдущего неравенства дает неравенстBо $(4.10)$.

Таким образом, в силу (4.9), (4.10) и (4.6) имеем $\langle K \boldsymbol{x}, \boldsymbol{x}\rangle \geqslant$ $\left\langle D x_{1}, x_{1}\right\rangle \geqslant 0$ для любого $x \in \mathbf{R}^{n+m}$. Это означает, что матрица (4.7) является ковариационной. Кроме того, взяв в последнем неравенстве минимум по $x_{0} \in \mathbf{R}^{n}$, получим неравенство $\left\langle\left(K_{\theta \theta}-K_{\theta \gamma} K_{\gamma \gamma}^{+} K_{\gamma \theta}\right) x_{1}, x_{1}\right\rangle \geqslant$ $\left\langle D x_{1}, x_{1}\right\rangle$, эквивалентное (4.8). Очевидно, что $\operatorname{cov}(\rho, \rho)=R$, т.е. матрица (4.7) удовлетворяет всем равенствам (4.4), так что $K \in \mathscr{K}$.

В силу (4.8) для этой матрицы $K$ выполнено неравенство $\operatorname{Sp} C^{\mathrm{T}}\left(K_{\theta \theta}-K_{\theta \gamma} K_{\gamma \gamma}^{+} K_{\gamma \theta}\right) C \geqslant \operatorname{Sp} C^{\mathrm{T}} D C$, поэтому значение задачи $(2.1)$ не меньше значения задачи (4.2). Формула (4.2) доказана.

Пусть теперь $D_{*}, T_{*}, R_{*}$ - решение задачи (4.2). Обозначим соответствуюшую матрицу (4.7) через $\bar{K}$. Тогда в силу предыдущего неравенства получим $\operatorname{Sp} C^{\mathrm{T}}\left(\bar{K}_{\theta \theta}-\bar{K}_{\theta \gamma} \bar{K}_{\gamma \gamma}^{+} \bar{K}_{\gamma \theta}\right) C \geqslant \operatorname{Sp} C^{\mathrm{T}} D_{*} C=D_{\min }$, т.е. $\bar{K}$ - решение задачи (2.1). Следовательно, по теореме 2.1 наименее благоприятным будет нормальное распределение вектора $(\theta, \rho)$ в случаe (4.3), и минимаксно-байесовская оценка будет линейна. Теорема 4.1 доказана. 
Следствие 4.1. Матрица условной ковариачии вектора $l=C^{\mathrm{T}} \theta$ относительно вектора $\gamma=A \theta+\rho$ для наименее благоприятного распределения $P_{*}$ равна $C^{\mathrm{T}} D_{*} C$.

Д о к а з а т е л ь с т в о. Обозначим через $\bar{D}$ матрицу (4.5), соответствующую матрице $\bar{K}$. Как мы только что показали, матрица $\bar{D}$ удовлетворяет ограничениям задачи (4.2) и неравенству $\bar{D} \geqslant D_{*}$. (см. (4.8)). Поэтому $C^{\mathrm{T}} \bar{D} C \geqslant C^{\mathrm{T}} D_{*} C$. В действительности здесь достигается равенство, ибо в противном случае мы получили бы, что $C^{\mathrm{T}} \bar{D} C=C^{\mathrm{T}} D_{*} C+U$, где $U \geqslant 0, U \neq 0$. Тогда $\operatorname{Sp} U>0$, и поэтому $\operatorname{Sp} C^{\mathrm{T}} \bar{D} C>\operatorname{Sp} C^{\mathrm{T}} D_{*} C$, т.е. $D_{*}$ - не решение задачи (4.2). Следствие 4.1 доказано.

Статистический смысл этого результата заключается в том, что матрица условной ковариации $C^{\mathrm{T}} D_{*} C$ вектора $l=C^{\mathrm{T}} \theta$ для наименее благоприятного распределения равна, грубо говоря, минимальной из ковариационных матриц

$$
D_{0}:=C^{\mathrm{T}} T_{*} C, \quad D_{1}:=C^{\mathrm{T}}\left(A^{\mathrm{T}} R_{*}^{-1} A\right)^{-1} C
$$

оценок вектора $l$, полученных из априорных данных $\theta_{0}$ и методом наименьших квадратов по измерениям $\gamma$ (поскольку отношение $D_{0} \geqslant D_{1}$ задает лишь частичный порядок на матрицах ковариаций, то упорядочивание производится относительно скалярной величины $\left.\mathbf{E}|\hat{l}-l|^{2}\right)$. Далее мы покажем, что если оценивается весь вектор параметров, то для получения минимаксной оценки в случае произвольной корреляции сигнала и шума нужно по одним направлениям взять МНК-оценки параметров, а по остальным довольствоваться априорными значениями этих параметров.

Случай известных ковариачий векторов $\theta$ и $\rho$. Здесь мы будем считать, что ковариационные матрицы векторов $\theta$ и $\rho$ нам точно известны, так что $\mathscr{P}=\mathscr{P}_{0}-$ класс всех распределений $P(y)$ случайного вектора $Y=(A \theta+\rho, \theta)$, удовлетворяющих (4.1) при $\mathscr{T}=\{T\}, \mathscr{R}=\{R\}, T=T_{*}$, $R=R_{*}$. По-видимому, даже в этом случае не существует простого выражения для гарантированной точности $D(\hat{l})$ на классе всех линейных оценок $\hat{l}(\gamma)$. Поэтому сначала мы рассмотрим задачу (1.2) на более узком классе оценок

$$
\hat{l}=Q \hat{l}_{1}+(I-Q) \hat{l}_{0}, \quad Q^{\mathrm{T}}=Q, \quad Q^{2}=Q,
$$

в которых $Q$ - это ортогональный проектор в $\mathbf{R}^{r}, \hat{l}_{0}=l_{0}=$ $C^{\mathrm{T}} \theta_{0}-$ априорная оценка вектора $l=C^{\mathrm{T}} \theta$, а $\hat{l}_{1}=C^{\mathrm{T}} \hat{\theta}_{1}=$ $C^{\mathrm{T}}\left(A^{\mathrm{T}} R_{*}^{-1} A\right)^{-1} A^{\mathrm{T}} R_{*}^{-1} \gamma-$ МНК-оценка этого вектора (см. (3.7)). Оказывается, что в этом случае величина апостериорной точности $\mathbf{E}_{P}|\hat{l}-l|^{2}$ будет одной и той же для всех распределений $P(y)$ с фиксированными $\operatorname{cov}(\theta, \theta), \operatorname{cov}(\rho, \rho)$ и произвольной взаимной ковариацией векторов $\theta$ и $\rho$. 
Теорема 4.2. Точность ортогональной оченки (4.12) не зависит от взаимной ковариачии случайных параметров $\theta$ и вектора ошибок $\rho$ :

$$
D(\hat{l}) \equiv \mathbf{E}_{P}|\hat{l}-l|^{2}=\operatorname{Sp}\left[Q D_{1}+\left(I_{r}-Q\right) D_{0}\right] \quad \forall P \in \mathscr{P}_{0},
$$

и оптимальной в таком классе будет оченка

$$
\hat{l}_{\perp}=Q_{\perp} \hat{l}_{1}+\left(I_{r}-Q_{\perp}\right) \hat{l}_{0},
$$

в которой $Q_{\perp}$ - это ортогональный проектор на положительное собственное пространство матричы $D_{0}-D_{1}$. Если $\propto$ тому же оченивается весь вектор параметров, т.е.

$$
\operatorname{rank} C=r=m,
$$

mo

$$
D\left(\hat{l}_{\perp}\right)=\sup _{D}\left\{\operatorname{Sp} C^{\mathrm{T}} D C: D \leqslant T, D \leqslant D(R)\right\},
$$

причем задача (4.16) имеет единственное решение $D_{\perp}$, определяемое равенством

$$
C^{\mathrm{T}} D_{\perp} C=Q_{\perp} D_{1}+\left(I_{m}-Q_{\perp}\right) D_{0} .
$$

Поясним, что если симметрическая матрица $H$ имеет собственные числа $\lambda_{j}=\lambda_{j}(H)$ и ортонормированные собственные векторы $p_{j}$, то ортогональный проектор на ее положительное собственное пространство равен

$$
Q_{\perp}=\sum_{j: \lambda_{j}>0} p_{j} p_{j}^{\mathrm{T}}
$$

Д ок аз а тельст в о. Поскольку $\hat{l}=l_{0}+X^{\mathrm{T}}\left(\gamma-\gamma_{0}\right)$, где $X^{\mathrm{T}}:=$ $Q C^{\mathrm{T}}\left(A^{\mathrm{T}} R^{-1} A\right)^{-1} A^{\mathrm{T}} R^{-1}=Q C^{\mathrm{T}} D(R) A^{\mathrm{T}} R^{-1}$, то

$$
\begin{aligned}
\mathbf{E}_{P}|\hat{l}-l|^{2}= & \left.\mathbf{E}_{P} \mid\left(A^{\mathrm{T}} X-C\right)^{\mathrm{T}} \theta+X^{\mathrm{T}} \rho\right)\left.\right|^{2}=\left\langle T\left(A^{\mathrm{T}} X-C\right), A^{\mathrm{T}} X-C\right\rangle \\
& +\langle R X, X\rangle+2 \operatorname{Sp} X\left(A^{\mathrm{T}} X-C\right)^{\mathrm{T}} \operatorname{cov}(\theta, \rho) .
\end{aligned}
$$

Отсюда следует, что эта величина не зависит от $\operatorname{cov}(\theta, \rho)$, ибо

$$
\begin{aligned}
X\left(A^{\mathrm{T}} X-C\right)^{\mathrm{T}} & =X(C Q-C)^{\mathrm{T}}=R^{-1} A D(R) C Q\left(Q-I_{r}\right) C^{\mathrm{T}} \\
& =R^{-1} A D(R) C\left(Q^{2}-Q\right) C^{\mathrm{T}}=0 .
\end{aligned}
$$

Так как обе матрицы $Q$ и $I_{r}-Q$ суть проекторы, то

$$
\begin{aligned}
\mathbf{E}_{P}|\hat{l}-l|^{2}= & \langle T(C Q-C), C Q-C\rangle+\langle R X, X\rangle=\operatorname{Sp} T C\left(Q-I_{r}\right)^{2} C^{\mathrm{T}} \\
& +\operatorname{Sp} A D(R) C Q^{2} C^{\mathrm{T}} D(R) A^{\mathrm{T}} R^{-1}=\operatorname{Sp} C^{\mathrm{T}} T C\left(I_{r}-Q\right) \\
& +\operatorname{Sp} C Q C^{\mathrm{T}} D(R)=\operatorname{Sp} C^{\mathrm{T}} T C\left(I_{r}-Q\right)+\operatorname{Sp} Q C^{\mathrm{T}} D(R) C,
\end{aligned}
$$

что с учетом обозначений (4.11) дает (4.13). 
Теперь из (4.13) и леммы 5.2 (см. также замечание 5.1) вытекает, что оценка (4.14) оптимальна на классе ортогональных оценок (4.12). При выполнении условия (4.15) стоящая в правой части (4.16) экстремальная задача эквивалентна задаче

$$
\sup _{D}\left\{\operatorname{Sp} D: D \leqslant D_{k}, k=0,1\right\}
$$

которая по лемме 5.3 имеет единственное решение, определяемое равенством (4.17). Равенство (4.16) следует из следствия 5.1 и замечания 5.1. Теорема 4.2 доказана.

С этого места до конца п. 4 предполагается выполненным условие (4.15).

Следствие 4.2. Eсли матрича $D_{\perp}$ в (4.17) неотричательно определена, то она дает решение двойственной задачи (4.2), а ортогональная оченка (4.14) дает решение исходной задачи (1.2).

Доказ ательство. В этом случае матрица $D_{\perp}$ удовлетворяет всем ограничениям двойственной задачи (4.2) и максимизирует функционал $\operatorname{Sp} C^{\mathrm{T}} D C$ даже на более широком множестве $\{D: D \leqslant T, D \leqslant D(R)\}$. Тогда по равенству (4.16) $D\left(\hat{l}_{\perp}\right)=\operatorname{Sp} C^{\mathrm{T}} D_{\perp} C=$ $D_{\min }$, т.е. оценка (4.14) дает решение исходной задачи (1.2).

Следствие 4.3. Если матричь (4.11) коммутируют, то ортогональная оченка (4.14) дает решение исходной задачи (1.2).

Доказательст во. Если $D_{0}$ и $D_{1}$ коммутируют, то они имеют общий набор ортонормированных собственных векторов $\left\{p_{j}\right.$, $j=1, \ldots, m\}$, совпадающий с набором таких векторов для матрицы $D_{0}-D_{1}$. Поэтому матрица (4.17) имеет тот же набор ортонормированных собственных векторов и принимает вид

$$
C^{\mathrm{T}} D_{\perp} C=\sum_{j=1}^{m} \min \left(\lambda_{j}\left(D_{0}\right), \lambda_{j}\left(D_{1}\right)\right) p_{j} p_{j}^{\mathrm{T}} .
$$

Эта матрица неотрицательно определена, так как $\lambda_{j}\left(D_{k}\right) \geqslant 0$, $j=1, \ldots, m, k=0,1$, поэтому матрица $D_{\perp}$ тоже неотрицательно определена. Следствие 4.3 доказано.

Стоит отметить, что в этом случае

$$
\hat{l}_{*}=\sum_{j \in \omega_{1}}\left\langle\hat{l}_{1}, p_{j}\right\rangle p_{j}+\sum_{j \in \omega_{0}}\left\langle\hat{l}_{0}, p_{j}\right\rangle p_{j}
$$

где $\omega_{1}$ - набор тех индексов, для которых собственные числа матрицы $D_{1}$ меньше соответствуюших собственных чисел матрицы $D_{0}, \mathbf{a} \omega_{0}$ его дополнение.

Следствие 4.4. Eсли $l=\theta$ и $T:=\operatorname{cov}(\theta, \theta)=\sigma^{2} I_{m}, m o$

$$
\hat{\theta}_{*}=\sum_{\lambda_{j}<\sigma^{2}}\left\langle\hat{\theta}_{1}, p_{j}\right\rangle p_{j}+\sum_{\lambda_{j} \geqslant \sigma^{2}}\left\langle\hat{\theta}_{0}, p_{j}\right\rangle p_{j}
$$


где $\lambda_{j}$ и $p_{j}, j=1, \ldots, m$, - это собственные числа и ортонормированные собственные векторы матричы $D_{1}=\left(A^{\mathrm{T}} R_{*}^{-1} A\right)^{-1}$. Наименее благоприятно нормальное распределение в случае $\operatorname{cov}(\rho, \theta)=-A D_{*} u$ матрицы

$$
D_{*}=\sum_{j=1}^{m} \min \left(\sigma^{2}, \lambda_{j}\left(D_{1}\right)\right) p_{j} p_{j}^{\mathrm{T}} .
$$

Сравнивая (4.16) с (4.2), легко заметить, что ортогональная оценка (4.14) будет оптимальной только в том случае, когда матрица $D_{\perp}$ неотрицательно определена. Но даже когда это не так, оказывается, что минимаксно-байесовская оценка имеет вид

$$
\hat{l}_{*}=Q_{*} \hat{l}_{1}+\left(I_{m}-Q_{*}\right) \hat{l}_{0}, \quad Q_{*}^{2}=Q_{*},
$$

на этот раз с неортогональным проектором $Q_{*}$. Следующий результат показывает, что для ее нахождения мы можем сначала вычислить ортогональные оценки $\hat{\mathbf{l}}_{\perp}$ для всех векторных параметров $1=V l, V \geqslant I_{m}$, а потом выбрать среди них самую лучшую.

Теорема 4.3. Исходная задача (1.2) сводится $\kappa$ задаче

$$
\inf _{V \geqslant I_{m}}\left\{D\left(\hat{\mathbf{l}}_{\perp}\right): \mathbf{l}:=V l\right\}
$$

т.е. а) значения задач (1.2) и (4.20) одинаковы; b) если $V_{*}$ - решение задачи (4.20), то минимаксно-байесовской будет линейная оченка $\hat{l}_{*}=V_{*}^{-1} \hat{\mathbf{l}}_{\perp}$, в которой $\hat{\mathbf{l}}_{\perp}=Q_{\perp} \hat{\mathbf{l}}_{1}+\left(I_{m}-Q_{\perp}\right) \hat{\mathbf{l}}_{0}-$ ортогональная оченжа (4.14) вектора $\mathbf{l}:=V_{*} l=V_{*} C^{\mathrm{T}} \theta, \hat{\mathrm{l}}_{1}:=V_{*} \hat{l}_{1}-$ МНК-оченка этого вектора, $\hat{\mathbf{l}}_{0}:=V_{*} \hat{l}_{0}=V_{*} C^{\mathrm{T}} \theta_{0}-$ его априорная оченка, $а Q_{\perp}-$ это ортогональный проектор на положительное собственное пространство матрицы $V_{*}\left(D_{0}-D_{1}\right) V_{*}$.

Д о к а з а т е л ь с т в о. Согласно (4.16), задачу (4.20) можно записать в таком виде: $\inf _{V \geqslant I_{m}} \sup _{D}\left\{\operatorname{Sp}(C V)^{\mathrm{T}} D(C V): D \leqslant T, D \leqslant D(R)\right\}$. По лемме 5.4 это выражение совпадает с правой частью (4.2), т.е. значения задач (1.2) и (4.20) одинаковы.

Пусть $V_{*}$ будет решением задачи $(4.20)$ и $\nu:=\hat{l}_{*}-l$. Тогда, согласно $(4.20)$ и $(4.13)$, получим

$$
\begin{aligned}
D_{\min } & =D\left(\hat{\mathbf{l}}_{\perp}\right) \equiv \mathbf{E}_{P}\left|\hat{\mathbf{1}}_{\perp}-\mathbf{l}\right|^{2}=\mathbf{E}_{P}\left|V_{*}\left(\hat{l}_{*}-l\right)\right|^{2} \\
& =\operatorname{Sp} \mathbf{E}_{P}\left[\left(V_{*} \nu\right)\left(V_{*} \nu\right)^{\mathrm{T}}\right]=\operatorname{Sp} V_{*}^{2} \mathbf{E}_{P}\left[\nu \nu^{\mathrm{T}}\right] \quad \forall P \in \mathscr{P}_{0} .
\end{aligned}
$$

Поскольку матрица $\mathbf{E}_{P}\left[\nu \nu^{\mathrm{T}}\right]$ неотрицательно определена и $V_{*}^{2} \geqslant I_{m}$, то из (4.21) получаем (см. [2])

$$
D_{\min }=\operatorname{Sp} V_{*}^{2} \mathbf{E}_{P}\left[\nu \nu^{\mathrm{T}}\right] \geqslant \operatorname{Sp} \mathbf{E}_{P}\left[\nu \nu^{\mathrm{T}}\right]=\mathbf{E}_{P}\left|\hat{l}_{*}-l\right|^{2} \quad \forall P \in \mathscr{P}_{0},
$$


и, следовательно, $D_{\min } \geqslant \sup _{P \in \mathscr{P}} \mathbf{E}_{P}\left|\hat{l}_{*}-l\right|^{2}:=D\left(\hat{l}_{*}\right) \geqslant D_{\min }$. Это означает, что $D_{\min }=D\left(\hat{l}_{*}\right)=D\left(\hat{\mathbf{l}}_{\perp}\right)$, т.е. $\hat{l}_{*}$ - решение исходной задачи $(1.2)$. Теорема 4.3 доказана.

3 а м е ч а н и е. Минимаксную оценку $\hat{l}_{*}$ можно представить в виде (4.19) с неортогональным проектором $Q_{*}=V_{*}^{-1} Q_{\perp} V_{*}$.

\section{5. Вспомогательные результаты.}

Лемма 5.1. Для любой симметрической матриць $C$

$$
\inf _{B \geqslant C}\langle A, B\rangle= \begin{cases}\langle A, C\rangle, & A \geqslant 0, \\ -\infty, & \text { иначе. }\end{cases}
$$

Д ок аз а т е л ь с т в о. Если $A \geqslant 0$, то (см. [2]) $\langle A, B\rangle \geqslant\langle A, C\rangle$. Иначе $\langle A \boldsymbol{x}, \boldsymbol{x}\rangle<0$ для некоторого вектора $\boldsymbol{x} \neq 0$, и для матриц $B=C+\alpha x x^{\mathrm{T}}$ получим $\langle B y, y\rangle=\langle C y, y\rangle+\alpha\langle x, y\rangle^{2} \geqslant\langle C y, y\rangle$ при всех $y$ и $\alpha>0$, т.е. $B \geqslant C$. В то же время $\langle A, B\rangle=\langle A, C\rangle+\alpha\langle A x, x\rangle \longrightarrow-\infty$ при $\alpha \rightarrow+\infty$.

Лемма 5.2. Если $D_{0}, D_{1}$ - симметрические матричы порядка $r$, mo

$$
\inf _{0 \leqslant Q \leqslant I_{r}} \operatorname{Sp}\left\{Q D_{1}+\left(I_{r}-Q\right) D_{0}\right\}
$$

достигается на ортогональном проекторе (4.18) на положительное собственное пространство матрицы $D_{0}-D_{1}$.

Док а з а т е л в с т в о. Пусть $D_{0}-D_{1}=O^{\mathrm{T}} \Lambda O$, где $O-$ ортогональная матрица, а $\Lambda:=\operatorname{diag}\left\{\lambda_{i}\right\}-$ диагональная матрица из собственных чисел матрицы $D_{0}-D_{1}$. Поскольку задача (5.1) равносильна максимизации функционала $\left\langle Q, D_{0}-D_{1}\right\rangle$ на множестве $0 \leqslant Q \leqslant I_{r}$, то после замены переменных $Q=O^{\mathrm{T}} U O$ она перейдет в задачу максимизации функционала

$$
\operatorname{Sp}\left(O^{\mathrm{T}} U O\right)\left(O^{\mathrm{T}} \Lambda O\right)=\operatorname{Sp} U \Lambda=\sum_{i=1}^{r} u_{i i} \lambda_{i}
$$

на множестве $0 \leqslant U \leqslant I_{r}$. Поскольку $0 \leqslant u_{i i} \leqslant 1, i=1, \ldots, r$, то максимум этого функционала по переменным $u_{i i}$ достигается при $u_{i i}=1$, если $\lambda_{i}>0$, и при $u_{i i}=0$, если $\lambda_{i} \leqslant 0, i=1, \ldots, r$. Положив $u_{i j}=0$ при $j \neq i$, получим диагональную матрицу $0 \leqslant U_{\perp} \leqslant I_{r}$ с диагональными элементами, равными нулю либо единице, которой соответствует ортогональный проектор $Q_{\perp}=O^{\mathrm{T}} U_{\perp} O$ на положительное собственное пространство матрицы $D_{0}-D_{1}$. Очевидно, что он и дает минимум в задаче (5.1).

3 а м е ч а н и е 5.1. Задача (5.1) имеет то же самое решение и на множестве всех ортогональных проекторов, которое вложено в множество $0 \leqslant Q \leqslant I_{r}$. 
Лемма 5.3. Если $D_{0}, D_{1}$ - симметрические матричы порядка $r$, mо задача

$$
\sup _{D}\left\{\operatorname{Sp} D: D \leqslant D_{k}, k=0,1\right\}
$$

имеет единственное решение

$$
D_{\perp}=Q_{\perp} D_{1}+\left(I_{r}-Q_{\perp}\right) D_{0}
$$

в котором $Q_{\perp}$ - ортогональный проектор на положительное собственное пространство матричы $D_{0}-D_{1}$.

Д ок аз а т е л ь с в о. Воспользуемся обозначениями, введенными в лемме 5.2. Поскольку след инвариантен относительно ортогональных преобразований, то после замены переменных $D=D_{0}-O^{\mathrm{T}} U O$ задача (5.2) перейдет в задачу максимизации функционала $\operatorname{Sp} D=$ $\operatorname{Sp} D_{0}-\operatorname{Sp} U=\operatorname{Sp} D_{0}-\sum_{i=1}^{r} u_{i i}$ на множестве всех симметрических матриц $U$ порядка $r$, которые удовлетворяют ограничениям

$$
U \geqslant 0, \quad U \geqslant \Lambda
$$

В частности, из этих ограничений вытекает, что $u_{i i} \geqslant 0, u_{i i} \geqslant \lambda_{i}$, $i=1, \ldots, r$, и поэтому $\sum_{i=1}^{r} u_{i i} \geqslant \sum_{i=1}^{r} \max \left(0, \lambda_{j}\right)$ для любой матрицы $U$, удовлетворяющей ограничениям (5.4).

Здесь равенство достигается только при $u_{i i}=\max \left(0, \lambda_{i}\right)$, $i=1, \ldots, r$. Так как $U \geqslant 0$, то если $u_{i i}=0$, то $u_{i j}^{2} \leqslant u_{i i} u_{j j}=0$, т.е. $u_{i j}=0$ при $j \neq i$. Аналогично, так как $U-\Lambda \geqslant 0$, где $\Lambda$ - диагональная матрица, то если $u_{i i}=\lambda_{i}$, то $\left(u_{i j}-0\right)^{2} \leqslant\left(u_{i i}-\lambda_{i}\right)\left(u_{j j}-\lambda_{j}\right)=0$, т.e. $u_{i j}=0$ при $j \neq i$. Это означает, что $U-$ диагональная матрица. Таким образом, матрица $D_{\perp}=D_{0}-O^{\mathrm{T}}\left[\operatorname{diag}\left\{\max \left(0, \lambda_{i}\right)\right\}\right] O=D_{0}-Q_{\perp}\left(D_{0}-D_{1}\right)$ дает единственное решение задачи (5.2). Лемма 5.3 доказана.

Следствие 5.1. Значения задач (5.1) u (5.2) совпадают.

Лемма 5.4. Если $D_{k} \geqslant 0$ nрu $k=0,1$, mо

$$
\begin{aligned}
& \max _{D \geqslant 0}\left\{\operatorname{Sp} D: D \leqslant D_{k}, k=0,1\right\} \\
& \quad=\inf _{V \geqslant I_{m}} \sup _{D}\left\{\operatorname{Sp} V D V: D \leqslant D_{k}, k=0,1\right\} .
\end{aligned}
$$

Eсли же $D_{k}>0, k=0,1$, то обе задачи (5.5) и (5.6) имеют решения $D_{*}$ $u V_{*}$, которые удовлетворяют условиям

$$
V_{*} \geqslant I_{m}, \quad V_{*} D_{*}=D_{*}=Q_{*} D_{1}+\left(I_{m}-Q_{*}\right) D_{0} \geqslant 0,
$$

где проектор $Q_{*}$ имеет вид $Q_{*}=V_{*}^{-1} Q_{\perp} V_{*}, a Q_{\perp}$ - это ортогональный проектор на положительное собственное пространство матричы $V_{*}\left(D_{0}-D_{1}\right) V_{*}$. И наоборот, если некоторые матричы $D_{*} u V_{*}$ удовлетворяют условиям (5.7), то $D_{*}$ - решение задачи (5.5), a $V_{*}$ - решение задачи (5.6). 
Д ок аз а те льс т в о. Поскольку $\operatorname{Sp} V D V=\operatorname{Sp} D V^{2}=\operatorname{Sp} D B$, где $B:=V^{2} \geqslant I_{m}$, то (5.6) можно записать как

$$
\inf _{B \geqslant I_{m}} \sup _{D}\left\{\operatorname{Sp} B D: D \leqslant D_{k}, k=0,1\right\} .
$$

По теореме о минимаксе [4] эта величина равна

$$
\sup _{D \leqslant D_{k}, k=0,1} \inf _{B \geqslant I_{m}} \operatorname{Sp} B D
$$

что с учетом леммы 5.1 дает значение задачи (5.5).

Ясно, что ограничения задачи (5.5) образуют компактное множество. Поэтому задача (5.5), так же как и задача (5.9), имеет решение. Покажем, что если $D_{k}>0, k=0,1$, то задачи (5.6) и (5.8) также имеют решения. При достаточно малых $\varepsilon>0$ матрица $D=\varepsilon I_{m}$ удовлетворяет ограничениям $D \leqslant D_{k}, k=0,1$. Кроме того, $\operatorname{Sp} B D=\varepsilon \operatorname{Sp} B>0$, если $B \geqslant 0, B \neq 0$. Поэтому выпуклая функция

$$
\varphi(B):=\sup _{D}\left\{\operatorname{Sp} B D: D \leqslant D_{k}, k=0,1\right\}
$$

положительна и конечна на множестве $B \geqslant 0, B \neq 0$. Следовательно, она непрерывна при $B>0[6]$.

Остается проверить, что ее множество уровня $\left\{B \geqslant I_{m}: \varphi(B) \leqslant\right.$ $\left.\varphi\left(I_{m}\right)\right\}$ ограничено. Предположим противное. Поскольку это множество выпукло и замкнуто, то оно содержит целый луч [6] $\left\{B: B=I_{m}+\right.$ $\alpha A, \alpha \geqslant 0\}$, где $A \geqslant 0, A \neq 0$. Тогда

$$
\varphi\left(I_{m}\right) \geqslant \varphi\left(I_{m}+\alpha A\right)=\alpha \varphi\left(\frac{I_{m}}{\alpha}+A\right) .
$$

Поскольку функция $\varphi(B)$ полунепрерывна снизу (как верхняя грань линейных функций), то $\lim _{\alpha \rightarrow \infty} \varphi\left(I_{m} / \alpha+A\right) \geqslant \varphi(A)>0$, что противоречит $(5.11)$, ибо тогда $\varphi\left(I_{m}\right) \geqslant+\infty$.

Таким образом, двойственные задачи (5.8), (5.9) имеют решения $B_{*}$ и $D_{*}$, которые образуют седловую точку [10], и поэтому по лемме 5.1 удовлетворяют условиям

$$
\begin{aligned}
\operatorname{Sp} B_{*} D_{*} & =\min _{B \geqslant I_{m}} \operatorname{Sp} B D_{*}=\operatorname{Sp} D_{*} \\
\operatorname{Sp} B_{*} D_{*} & =\max _{D \leqslant D_{k}, k=0,1} \operatorname{Sp} B_{*} D \\
& =\max _{D}\left\{\operatorname{Sp} V_{*} D V_{*}: V_{*} D V_{*} \leqslant V_{*} D_{k} V_{*}, k=0,1\right\}
\end{aligned}
$$

По лемме 5.3 из (5.13) получаем

$$
V_{*} D_{*} V_{*}=Q_{\perp}\left(V_{*} D_{1} V_{*}\right)+\left(I_{m}-Q_{\perp}\right)\left(V_{*} D_{0} V_{*}\right) \geqslant 0 .
$$

Умножая это равенство слева и справа на матрицу $V_{*}^{-1}$, получим второе из равенств (5.7). 
Далее, поскольку $B_{*} \geqslant I_{m}, D_{*} \geqslant 0$ и, согласно (5.12), $\operatorname{Sp}\left(B_{*}-\right.$ $\left.I_{m}\right) D_{*}=0$, то $\left(B_{*}-I_{m}\right) D_{*}=0[2]$, т.е.

$$
\left(V_{*}+I_{m}\right)\left(V_{*}-I_{m}\right) D_{*}=\left(V_{*}^{2}-I_{m}\right) D_{*}=0 \text {, }
$$

что дает равенство $V_{*} D_{*}=D_{*}$.

Наоборот, пусть выполнены условия (5.7). Тогда выполнены и соотношения $(5.14),(5.15)$, т.е. $B_{*}$ - решение задачи $(5.12)$, а $V_{*} D_{*} V_{*}$ решение (5.13). Последнее удовлетворяет ограничениям в $(5.13)$, поэтому $D_{*} \leqslant D_{k}, k=0,1$. Иными словами, $\left(B_{*}, D_{*}\right)$ - седловая точка в задаче (5.8). Тогда $B_{*}$ - решение задачи (5.8), а $D_{*}$ - решение задачи (5.9) (см. [10]), а тем самым и задачи (5.5). Лемма 5.4 доказана.

\section{СПИСОК ЛИТЕРАТУРЫ}

1. Алберт A. Регрессия, псевдоинверсия и рекуррентное оценивание. М.: Наука, 1977, 224 c.

2. Беллман Р. Введение в теорию матриц. М.: Мир, 1969, 352 с.

3. Липчер P. Ш., Ширяев А. Н. Статистика случайных процессов. М.: Наука, 1974, $696 \mathrm{c}$.

4. Обен Ж. П., Экланд И. Прикладной нелинейный анализ. М.: Мир, 1988, 512 с.

5. Панжов A.P. Рекуррентная условно-минимаксная фильтрапия процессов в разностных нелинейных стохастических системах. - Техн. киберн., 1992, № 3, с. 6370.

6. Рокафеллар P. T. Выпуклый анализ. М.: Наука, 1973, 471 с.

7. Соловьев $B . H$. Минимаксное и минимаксно-байесовское оценивание на классах распределений с ограниченными вторыми моментами. - Успехи матем. наук, 1995 , т. 50, № 4 , с. 171-172.

8. Соловьев $B . H$. Минимаксно-байесовское оценивание параметров линейной регрессии. - Успехи матем. наук, 1996, т. 51, № 3, с. 211-212.

9. Соловьев $B . H$. Двойственные экстремальные задачи и их применения к задачам минимаксного оценивания. - Успехи матем. наук, 1997, т. 52, № 4, с. 49-86.

10. Экланд И., Темам Р. Выпуклый анализ и вариационные проблемы. М.: Мир, $1979,400 \mathrm{c}$.

11. Berger J. O. Statistical Decision Theory and Bayesian Analysis. New York: SpringerVerlag, 1985.

12. Berger J.O. Robust Bayesian analysis: sensitivity to the prior. - Statist. Plann. Inference, 1990, v. 25, p. 303-328.

13. Lauter $H$. A minimax linear estimator for linear parameters under restrictions in form of inequalities. - Math. Operations-forsch. und Statist., 1975, B. 6, № 5, S. 689-695.

14. Rao C. R. Linear Statistical Inference and Its Applications. New York: Wiley, 1971.

15. Soloviov V. Minimax estimation and the least squares method. - Stochastics Stochastics Rep., 1993, v. 42, p. 209-223.

16. Vandelinde $V . D$. Robust properties of solutions to linear-quadratic estimation and control problems. - IEEE Trans. Automat. Control, 1977, v. 22, № 1, p. 138-139.

17. Соловьев $B . H . \mathrm{K}$ вопросу о минимаксно-байесовском оценивании. - Успехи матем. наук, 1998, т. 53, № 5, с. 247-248. 